\title{
Photoplethysmographic waves and their detailed pulse interval distribution analysis on Poincare plots before and after the sauna exposures
}

Huotari, Matti, Määttä, Kari, Röning, Juha

Matti Huotari, Kari Määttä, Juha Röning, "Photoplethysmographic waves and their detailed pulse interval distribution analysis on Poincare plots before and after the sauna exposures," Proc. SPIE 11585, Biophotonics-Riga 2020, 115850F (28 October 2020); doi: 10.1117/12.2582204

SPIE Event: Third International Conference Biophotonics Riga 2020, 2020, Riga, Latvia 


\title{
Photoplethysmographic waves and their detailed pulse interval distribution analysis on Poincare plots before and after the sauna exposures
}

\author{
Matti Huotari ${ }^{1}$, Kari Määttä², Juha Röning1 \\ ${ }^{1}$ University of Oulu, BISG, Oulu, Finland, \\ ${ }^{2}$ University of Oulu, CAS, Oulu, Finland \\ E-mails:(matti.huotari7@gmail.com, Kari.Maatta@oulu.fi, Juha.Roning@oulu.fi)
}

\begin{abstract}
Photoplethysmography (PPG) has proved to be valuable signal carrier information from many biomedical sources. Especially, sauna studies have shown that heart pulse inter beat intervals, heart rate variability (HRV), shape and morphology of PPG can provide much interesting information about arterial elasticity during and after a sauna exposure. These parameters are pulse amplitude, pulse width, and pulse to pulse beat intervals changes caused by the sauna exposure. In our preliminary study there is searched on heart pulse variability information and other hemodynamic parameters. However, there is not yet standard detailed calculation algorithms for these parameters. Merged Poincaré plots, or so called return maps, could give valuable information which is here at the first time noticed.
\end{abstract}

Keywords: Photoplethysmography, infrared LED, red LED, photosensitive diode, phase sensitive detection, heart rate variability, merged Poincaré plot, sauna exposure, heart rate recovery

\section{INTRODUCTION}

Photoplethysmographic (PPG) recordings are affected by physiological factors related to the transmission of the pulse wave through the vascular bed. The accuracy of the PPG pulse wave detection depends on the pulse morphology and the recording site. There is a need to define the accurate fiducial point for performing a pulse rate variability (PRV) analysis under non-stationary conditions based on different PPG sensor locations, finger or toe during sauna exposures (SE). That's why to show that the existence of the wave length components is consistent and repeatable. However, our study would involve more subjects as the PPG signals registered from the finger with the RED and INFRARED LED lights. The PPG RED and INFRARED light measured from the finger differ from each other on the amplitude, but not much on the inter beat interval between the maxima of the second derivative PPG (SDPPG) waves. The large deviations, however, appear on a few cases. PPG measurements in rest it are easily done. PPG pulse series can be analyzed using the merged Poincaré plot analysis. This analysis was extended to evaluate PRV as a surrogate of heart rate variability (HRV) which can change by a SE. Various fields of medical technology use the quantification of HRV as an indicator of autonomic nervous system function, but have not recognized the importance of using standardized methods for the analysis of heart rate variability. The purpose of this study was to evaluate the effect of sauna exposure on HRV indicator values on the Poincaré plots. High levels of HRV values are generally signs of healthy efficient autonomic mechanisms of sympathetic and parasympathetic neural systems that characterize individuals like sport people. Low or reduced HRV values often shows an autonomic nervous system malfunction and may predict becoming health problems. The most relevant fiducial points are found is the first wave a of the second derivative of PPG waves (SDPPG) ${ }^{1}$. In practice, Poincaré plots are an intuitive and commonly used nonlinear time based method to assess complex biosystems and biosignals. Our preliminary results demonstrated that the combination of two or more merged Poincaré plots could show promise as a method for distinguishing between different vascular functions. Using the merged Poincaré plots, both PPG and SDPTG technologies which are in many medical devices that are available in many hospital clinics. The ability of measuring HRV, blood oxygen saturation, blood pressure, cardiac output, and blood stroke volume for assessing autonomic function, detecting peripheral vascular disease, and predicting the risk of atherosclerosis reflecting the importance of these techniques. Because atherosclerosis disease is not homogeneous phenomena, it can depend on the blood viscosity. On the other hand, viscosity depends on red blood cells, their membrane elasticity, other blood molecules, and body temperature.

Biophotonics-Riga 2020, edited by Janis Spigulis, Proc. of SPIE Vol. 11585,

(C) 2020 SPIE · CCC code: 0277-786X/20/\$21 · doi: 10.1117/12.2582204 


\section{MATERIALS AND METHODOLOGY}

\subsection{Photoplethysmography}

PPG sensors are based on LEDs, photosensitive detector, and optoelectronic amplifier circuit. It makes possible to consider the origins of the PPG waveform characteristics. Finger and toe pulse waves are measured by 660 (red) \& 940 (infrared) $\mathrm{nm}$ in the PPG transmittance probes based on phase sensitive detection (PSD) at $1 \mathrm{kHz}$ sampling frequency.

\subsection{Subjects under study}

The studies was approved by the ethical review boards of the Oulu University, Oulu University Hospital and the Finnish National Supervisory Authority of Health and Welfare (VALVIRA). Informed consents were obtained from the test subjects, who abstained from alcohol, caffeine, and strenuous exercise in the $24 \mathrm{~h}$ up to the day of the tests. We recorded on 25 healthy subjects ( 4 females +21 males) with a mean age of $43.04 \pm 14.62$ S.D. years without known cardiovascular disorders. In the Table 1 is listed and shown in the Figure 3. In practice, the PPG measurement protocol was as follows: the subject was measured before the sauna exposure in the dressing room at the $22 \mathrm{oC}$. There was no delay after the sauna exposure before the measurement was taken immediately. There was no indication that the increased body temperature would affect the performance of the PPG sensors according to the followed measurement

\section{RESULTS}

For the comparison of the red and infrared PPG pulse wave in the merged Poincaré plots are shown in Figure 1 for a 31 years male person. When the triangles are merged, then they form a star, as in Figure 1. In the Figure 1 Poincaré plot is shown.

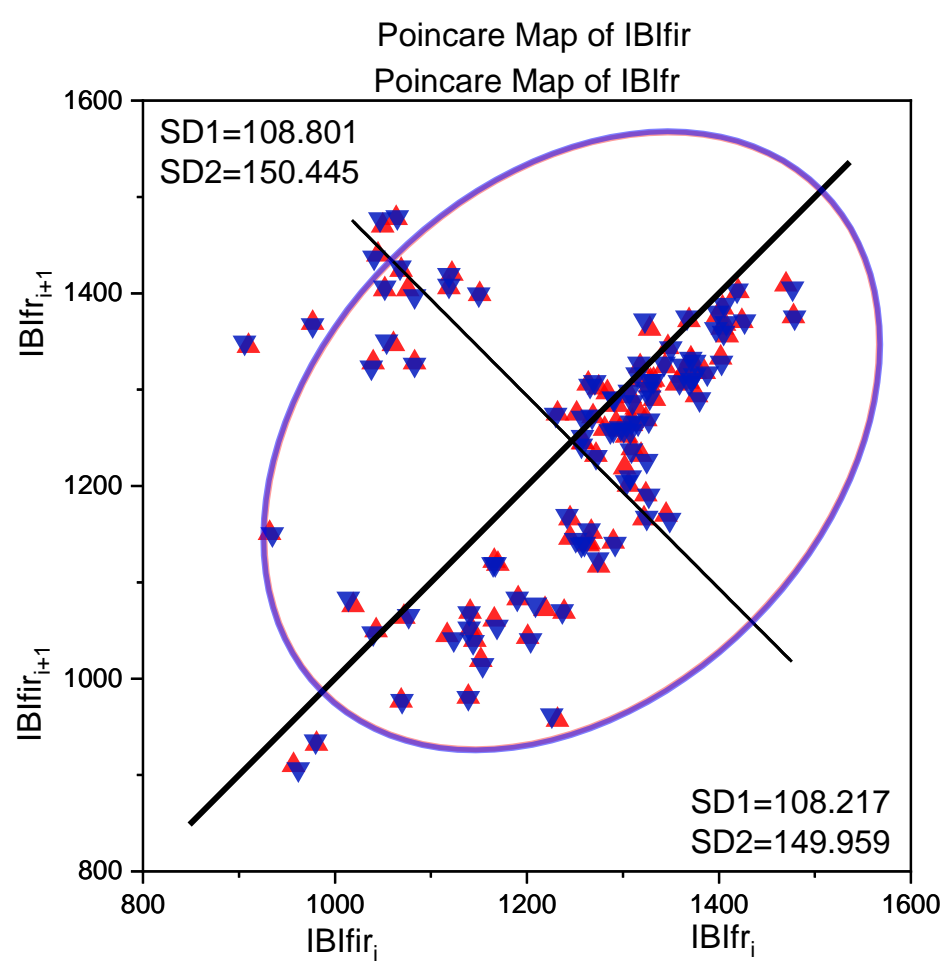

Figure 1. An example of a merged Poincare plot containing the infrared (red) and red (blue) IBIi vs IBIi+1 values for a 32 years male subject before the SE. The Poincare parameters (SD1 \& SD2 and a fitted ellipse) are shown for the subject's finger (red fr \& infrared fir). The comparison of the red and infrared PPG pulse wave in the merged Poincaré plot is constructed so that the infrared PPG pulse inter beat intervals (IBIfir) are shown by a red triangle pointing up, and the red PPG pulses, IBIfr, are marked by a blue triangle pointing down. The unit of the both axis is $1 \mathrm{~ms}(=1 / 1 \mathrm{kHz})$. 


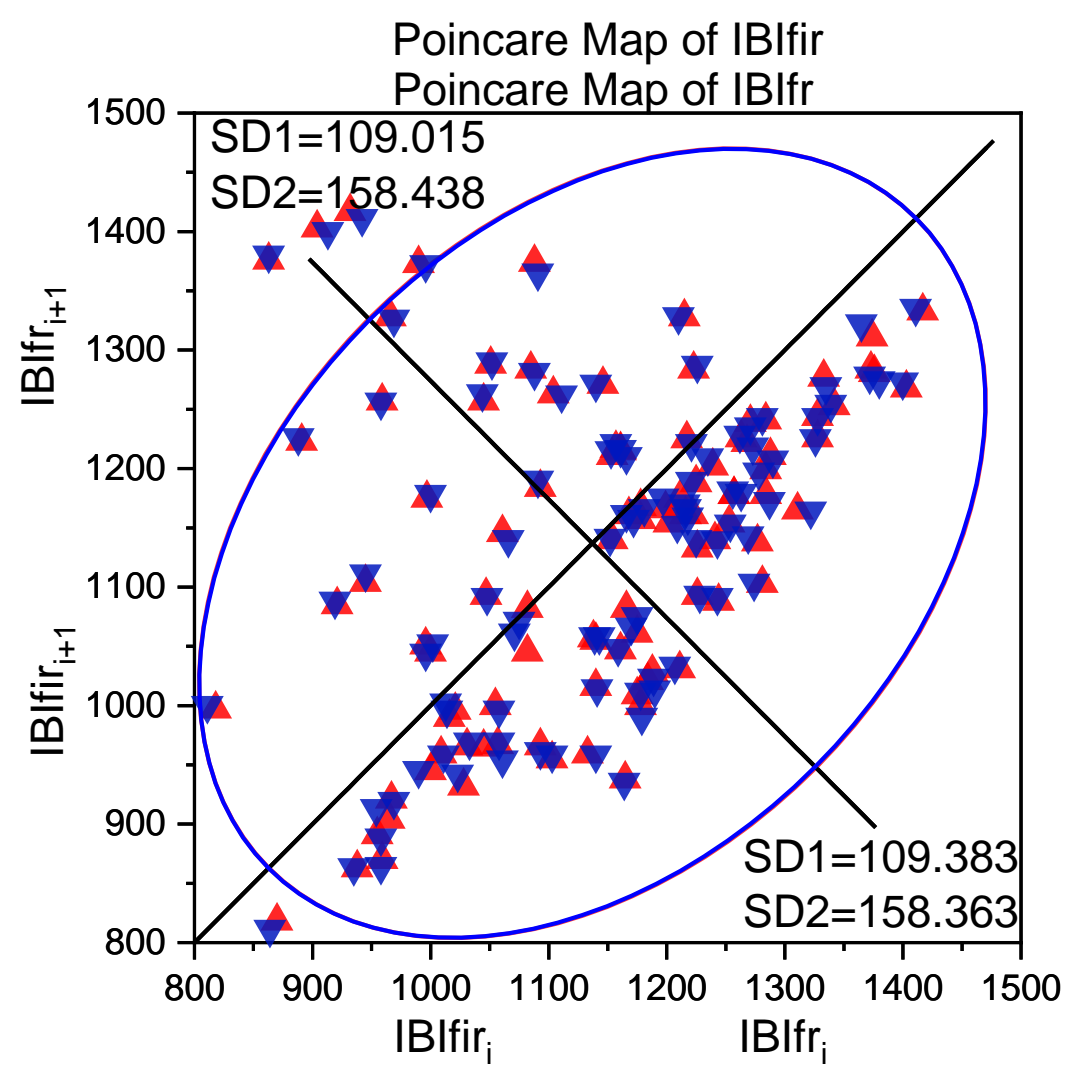

Figure 2. An example of a merged Poincare plot containing also the infrared (red) and red (blue) IBI $_{\mathrm{i}}$ vs IBI $_{\mathrm{i}+1}$ values for a 32 years male subject after the SE. The Poincare parameters (SD1 \& SD2 and a fitted ellipse) are shown for the subject's finger (red fr \& infrared fir). The comparison of the red and infrared PPG pulse wave in the merged Poincaré plot is constructed as in the Figure 1. The unit of the both axis is $1 \mathrm{~ms}$.

Table 1. The results (SD1 (ms), SD2 (ms), the area $\left(\mathrm{ms}^{2}\right)$ of ellipse before (bs) and after (as) SE, 25 test persons $(\mathrm{G}=\mathrm{gender})$.

\begin{tabular}{|l|l|l|l|l|l|l|l|l|l|}
\hline $\mathrm{G}$ & SD1 & $\begin{array}{l}\text { SD2 } \\
\text { (bs) }\end{array}$ & SD1 & $\begin{array}{l}\text { SD2 } \\
\text { (as) }\end{array}$ & $\begin{array}{l}\text { SD1/ } \\
\text { SD2 }\end{array}$ & $\begin{array}{l}\text { SD2/ } \\
\text { SD1 }\end{array}$ & $\begin{array}{l}\text { Diffe } \\
\text { rence }\end{array}$ & Area1 (bs) & $\begin{array}{l}\text { Area2 } \\
\text { (as) }\end{array}$ \\
\hline $\mathrm{m}$ & 9.266 & 37.642 & 6.054 & 36.166 & 0.246 & 0.167 & -0.078 & 1095.20 & 687.500 \\
\hline $\mathrm{m}$ & 54.195 & 99.425 & 13.807 & 51.158 & 0.545 & 0.270 & -0.275 & 16919.38 & 2217.900 \\
\hline $\mathrm{m}$ & 99.020 & 130.190 & 133.560 & 162.084 & 0.760 & 0.824 & 0.06345 & 40479.661 & 67974.530 \\
\hline $\mathrm{m}$ & 32.609 & 47.820 & 8.645 & 13.348 & 0.682 & 0.648 & -0.034 & 4896.398 & 362.330 \\
\hline $\mathrm{m}$ & 21.212 & 30.360 & 9.383 & 16.400 & 0,699 & 0.572 & -0.127 & 2022.148 & 483.190 \\
\hline $\mathrm{f}$ & 28.403 & 69.543 & 10.575 & 17.465 & 0.408 & 0.605 & 0.197 & 6202.222 & 579.930 \\
\hline $\mathrm{m}$ & 8.031 & 19.701 & 12.834 & 21.724 & 0.408 & 0.591 & 0.183 & 496.807 & 875.450 \\
\hline $\mathrm{m}$ & 80.827 & 157.932 & 17.721 & 44.068 & 0.512 & 0.402 & -0.110 & 40082.633 & 2452.117 \\
\hline $\mathrm{m}$ & 17.526 & 52.916 & 17.342 & 42.964 & 0.331 & 0.404 & 0.072 & 2912.054 & 2339.556 \\
\hline $\mathrm{m}$ & 8.986 & 27.830 & 5.178 & 26.653 & 0.323 & 0.194 & -0.129 & 785.252 & 433.350 \\
\hline $\mathrm{m}$ & 28.900 & 34.427 & 14.616 & 18.266 & 0.839 & 0.800 & -0.039 & 3124.112 & 838.313 \\
\hline $\mathrm{m}$ & 6.865 & 16.503 & 1.968 & 6.042 & 0.416 & 0.326 & -0.090 & 355.740 & 37.337 \\
\hline
\end{tabular}




\begin{tabular}{|l|l|l|l|l|l|l|l|l|l|}
\hline $\mathrm{m}$ & 15.786 & 51.892 & 7.656 & 20.600 & 0.304 & 0.372 & 0.067 & 2572.185 & 495.221 \\
\hline $\mathrm{m}$ & 37.136 & 40.709 & 25.277 & 46.529 & 0.912 & 0.543 & -0.369 & 4746.956 & 3692.996 \\
\hline $\mathrm{m}$ & 12.249 & 34.653 & 15.866 & 29.065 & 0.353 & 0.546 & 0.192 & 1332.819 & 1447.996 \\
\hline $\mathrm{m}$ & 26.691 & 65.179 & 4.961 & 29.507 & 0.409 & 0.16813 & -0.241 & 5462.635 & 459.646 \\
\hline $\mathrm{m}$ & 33.005 & 71,04 & 21.995 & 53.459 & 0.465 & 0.411 & -0.053 & 7362.280 & 3692.108 \\
\hline $\mathrm{m}$ & 29.835 & 61.838 & 31.881 & 69.894 & 0.482 & 0.456 & -0.026 & 5793.101 & 6996.832 \\
\hline $\mathrm{m}$ & 9.504 & 25.873 & 20.239 & 60.783 & 0.367 & 0.333 & -0.034 & 772.117 & 3862.788 \\
\hline $\mathrm{m}$ & 46.610 & 154.907 & 26.727 & 45.340 & 0.301 & 0.589 & 0.289 & 22671.476 & 3805.059 \\
\hline $\mathrm{m}$ & 26.727 & 45.340 & 2.801 & 9.510 & 0.589 & 0.295 & -0.295 & 3805.059 & 83.646 \\
\hline $\mathrm{f}$ & 39.526 & 80.174 & 10.575 & 17.4645 & 0.493 & 0.605 & 0.112 & 9950.50216 & 579.914 \\
\hline $\mathrm{f}$ & 10.198 & 30.9926 & 3.6271 & 12.1716 & 0.32905 & 0.298 & -0.031 & 992.43636 & 138.623 \\
\hline $\mathrm{f}$ & 16.819 & 17.149 & 14.133 & 15.658 & 0.981 & 0.903 & -0.078 & 905.646 & 694.889 \\
\hline $\mathrm{m}$ & 33.658 & 58.542 & 16.8425 & 33.469 & 0.57493 & 0.503 & -0.072 & 6187.051 & 1770.007 \\
\hline
\end{tabular}

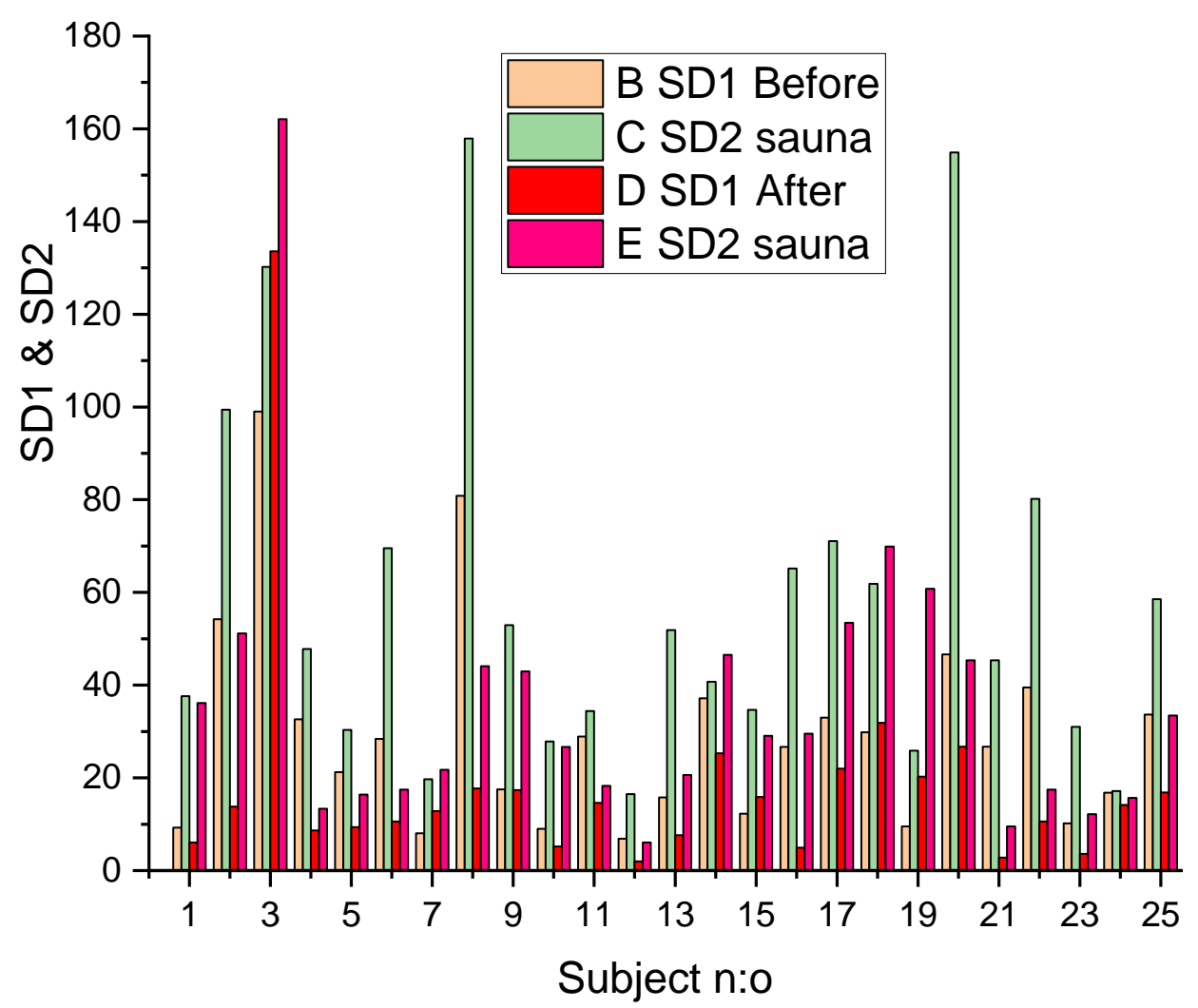

Figure 3. The results of SD1 (ms) (yellow, red) and SD2 (ms) (green, redish) of the ellipse before and after SE, 25 subjects. 


\section{DISCUSSIONS}

The Poincare plot indexes (SD1 \& SD2) of IBI are able to quantify and monitor shifts of sympathovagal balance toward sympathetic dominance induced by a single $30 \mathrm{~min} \mathrm{SE}$ at $80^{\circ} \mathrm{C}$ temperature. Poincare plots are also effective in detecting errors like ectopic beats, or other rhythm abnormalities. ${ }^{3,4,5,6}$ Quantitative analysis of Poincare plot is typically performed by fitting an ellipse to the plot of $\mathrm{IBI}_{\mathrm{i}} \mathrm{vs}_{\mathrm{IBI}} \mathrm{i}_{+1}$. Poincare plot analysis is based that one axis is at 45 degrees according to the line-of-identity, $\mathrm{IBI}_{\mathrm{i}}=\mathrm{IBI}_{\mathrm{i}+1}$. The standard deviation of the points in the direction perpendicular to the line-of-identity is the term SD1, and it is a measure of an instantaneous HRV. On the other hand, the standard deviation of the IBI points in the parallel to the line-of-identity is the term SD2 and it is a measure of longer term HRV. The clinical utility of PPG based Poincaré plot analysis could indicate health impairment or predicting a healthy heart. ${ }^{7}$ In healthy subjects, the HRV is predominantly affected by the sympathetic and parasympathetic nervous system by various stimuli, e.g., temperature. However, further investigations using larger subject number to determine even more variabilities in the vital health-related information. In the SE, dehydration could be contributors to the elevated of blood viscosity. It is very interesting that the merged Poincare plots (infrared and red) of the sport people have the HRV points overlapping, whereas the elderly people seldom have overlapping points. Blood flow in the peripheral arteries is influenced by both viscosity and hematocrit. In addition, the authors believe that the overlapping between the parameters IBIfir and IBIfr are caused by physiological health reasons, and not by noise of the measuring system nor software made mistakes. However, many challenges remain with the technology, according to the literature ${ }^{2}$.

\section{REFERENCES}

[1] Y. K.Qawqzeh, R. Uldis, and M. Alharbi, (2015) "Photoplethysmogram second derivative review: Analysis and applications,” Academic Journals Vol. 10(21), pp. 633-639.

[2] D. Castaneda, A. Esparza, M. Ghamari, C. Soltanpur, H. Nazeran, (2018) “A review on wearable photoplethysmography sensors and their potential future applications in health care", Int $\mathrm{J}$ Biosens Bioelectron. 4(4): 195-20.

[3] M. Imamura, S. Biro, T. Kihara, S. Yoshifuku, K. Takasaki, Y. Otsuji, S. Minagoe, Y. Toyama, C. Tei (2001) Repeated Thermal Therapy Improves Impaired Vascular Endothelial Function in Patients With Coronary Risk Factors. Journal of the American College of Cardiology, 38, 4.

[4] T. Kihara, S. Biro, M. Imamura, S. Yoshifuku, K. Takasaki, Y. Ikeda, Y. Otuji, S. Minagoe, Y. Toyama, C. Tei (2002) Repeated Sauna Treatment Improves Vascular Endothelial and Cardiac Function in Patients With Chronic Heart Failure. Journal of the American College of Cardiology, 39, 5.

[5] A.M. Cataia, C.M. Pastre, M.F. de Godoy, E. da Silva, A.C.M Takahashi, L.C.M. Vanderlei (2020): Heart rate variability: are you using it properly? Standardization checklist of procedures. Brazilian Journal of Physical Therapy; 24(2):pp91-102.

[6] A. Choi, H. Shin (2018) Quantitative Analysis of the Effect of an Ectopic Beat on the Heart Rate Variability in the Resting Condition. Front. Physiol. 9:922

[7] R. Sassi, S. Cerutti, F. Lombardi, M. Malik, H. V. Huikuri, C.-K. Peng, G. Schmidt, Y. Yamamoto (2015) Advances in heart rate variability signal analysis: joint position statement by the e-Cardiology ESC Working Group and the European Heart Rhythm Association co-endorsed by the Asia Pacific Heart Rhythm Society, Europace 17, 13411353. 